\title{
Modeling of star formation histories of dwarf ellipticals
}

\author{
N. Arimoto \\ National Astronomical Observatory of Japan, Mitaka, Tokyo 181-8588, Japan \\ email: arimoto@optik.mtk.nao.ac.jp
}

\begin{abstract}
Cosmological numerical simulations suggest that dSphs and dEs formed hierarchically via assembling much smaller stellar/gas clumps. Star formation had occurred intensively during the building up phase that is shorter than $\sim 1$ Gyr. Dark matter assembled hierarchically, while gas collapsed dissipatively; as a result the metallicity gradient was built up and stars at the galaxy centre tend to have larger rotational velocity and smaller velocity dispersion. There are increasing evidences, however, that suggest rather long-lasted star formation in dwarf galaxies with much smaller SFRs, with which cosmological simulations need to be reconciled.
\end{abstract}

Keywords. galaxies: abundances, galaxies: dwarf, galaxies: elliptical and lenticular, cD, galaxies: evolution, galaxies: formation, (galaxies:) Local Group, galaxies: stellar content

\section{Introduction}

Star formation histories of dwarf elliptical galaxies (dEs) are unknown. Classical scenarios suggested that a $\mathrm{dE}$ should have experienced an early intensive burst of star formation, which followed quickly by a galactic wind induced by supernovae explosions (eg, Yoshii \& Arimoto (1988)). Thus, in short, star formation in a dE is intensive and short. As a result, a dE is composed of old and metal-poor stars whose chemical abundances are similar to the yield patterns of type II supernovae (SNeII). There is an increasing evidence, however, suggesting that dEs might have experienced long lasting star formation with moderately low star formation rate (SFR). For example, metal-poor RGB stars $([\mathrm{Fe} / \mathrm{H}] \leqslant-2)$ of dwarf spheroidals (dSphs) in the Local Group have much lower $[\alpha / F e]$ ratios compared to the equivalents of Galactic halo (eg, Shetrone et al. (2001)). Spectroscopic analyses of M32, a compact $\mathrm{dE}$ in the Local Group, have shown that this galaxy has young/intermediate age stellar populations at least at the galaxy centre (eg, Rose et al. (2005)). dSphs in the Local Group often show narrow RGBs in spite of the fact that RGB stars show a wide spread in metallicities, which implies that metal-rich stars are much younger than metal-poor ones (Ikuta \& Arimoto (2002)), ie, star formation lasted much longer than expected by a classical monolithic collapse scenario.

Are dwarf galaxies building blocks of more massive ones? Recent Subaru (SuprimeCam) imaging of Leo A, a dwarf irregular galaxy, has shown that this very small galaxy has an old and metal-poor stellar halo (Vansevicius et al. (2004)). This might indicate that even the smallest dwarf galaxies had been formed hierarchically.

Indeed, latest cosmological numerical simulations of $\mathrm{dSph} / \mathrm{dE}$ galaxies are successful in reproducing chemical and kinematical properties of dSph galaxies by building them up hierarchically, but these models always predict that stars formed intensively at the beginning of galaxy evolution and stopped well before the onset of type Ia supernovae (SNeIa), which apparently contradicts recently piling up evidences for long-lasting star formation. 


\section{Chemical Enrichment in dSph Galaxies}

The abundance patterns of the Draco, Sextans, and Ursa Minor dSphs all suggest that the chemical enrichment occurred with SFRs much lower than normal spiral galaxies (Ikuta \& Arimoto (2002)). Figure 1 reproduces the observed stellar abundances of the Draco, Sextans, and Ursa Minor dSphs in the $[\mathrm{Mg} / \mathrm{Fe}]-[\mathrm{Fe} / \mathrm{H}]$ diagram (Shetrone et al. (2001)). Despite the narrow widths of the RGB, the iron abundances exhibit a large dispersion $(-3 \leqslant[F e / H] \leqslant-1.4)$, and the abundance ratios of the $\alpha$-elements (e.g., $\mathrm{Mg})$ to iron are near or below solar. The abundance ratio $[\mathrm{Mg} / \mathrm{Fe}]$ starts to decrease at $[\mathrm{Fe} / \mathrm{H}] \simeq-2$. On the other hand, $[\mathrm{Mg} / \mathrm{Fe}]$ in the solar neighbourhood decreases with $[\mathrm{Fe} / \mathrm{H}]$ at around $[\mathrm{Fe} / \mathrm{H}] \geqslant-1$, which is generally interpreted as the onset of SNeIa explosions (e.g., Matteucci \& Greggio (1986)). Supposing that the decline of $[\mathrm{Mg} / \mathrm{Fe}]$ in the dSphs is also caused by SNeIa, the star formation should have continued longer than the lifetime (typically $1-2 \mathrm{Gyr}$ ) of progenitors of SNeIa and the SFRs should be much lower than that of the solar neighbourhood. A time-delay model (Matteucci \& Brocato (1990)) of the SNeIa precisely predicted that the low SFR results in lower $[\mathrm{Mg} / \mathrm{Fe}]$ ratios relative to stars in the Galaxy. Ikuta \& Arimoto (2002) have shown that the observed abundance patterns of these dSphs can best be reproduced by chemical evolution models in which SFR, initial mass function (IMF), and time duration of initial star formation are in the range of $\epsilon=0.01-0.005 \mathrm{Gyr}^{-1}$ (cf, $\epsilon_{\odot}=0.2 \mathrm{Gyr}^{-1}$ in the solar neighbourhood), the Salpeter IMF, and $\Delta T_{\mathrm{SF}}=3.9-6.5$ Gyrs, respectively. This star formation history can reproduce morphologies of the observed CMDs as well, such as narrow RGBs and red HBs and succeeds in solving the second parameter problem of the Draco dSph. Hence, both of the abundance patterns and the CMD morphologies can be explained by the star formation histories characterised by the low SFR and the long duration of the star formation period.

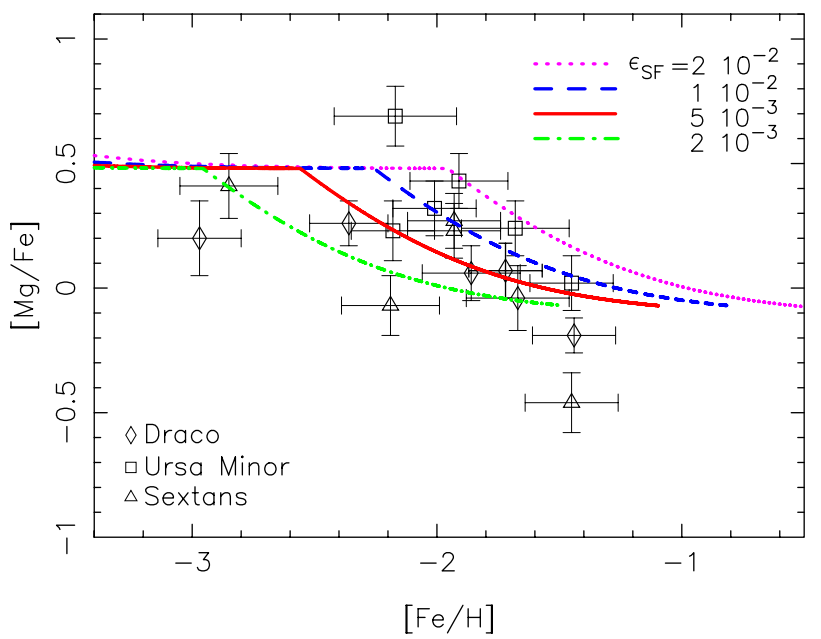

Figure 1. Theoretical abundance patterns together with the observations (Shetrone et al. (2001)) for stars in the Draco, Sextans, and Ursa Minor dSphs. The meaning of the marks is written on the panel. The same IMF (the Salpeter IMF: $\mathrm{x}=1.35)$ with upper $\left(m_{\mathrm{u}}=60 M_{\odot}\right)$ and lower $\left(m_{l}=0.1 M_{\odot}\right)$ mass limits are adopted in all the model. 


\section{Subaru/HDS Observation of dSph Ursa Minor}

Ikuta \& Arimoto (2002) have implicitely assumed that all dSphs should have almost identical star formation history which, however, might not be true judging from variety of CMD morphologies. Keeping this in our mind, we have started a campaign of Subaru/HDS observation of Ursa Minor dSph and have so far obtained high-resolution optical region spectra of 11 RGBs (Sadakane et al. (2004); Sadakane et al. (2005)). The mean stellar metallicities of $11 \mathrm{RGBs}$ is $\langle[\mathrm{Fe} / \mathrm{H}]\rangle=-2.0 \pm 0.3$ and many Ursa Minor RGBs show significantly low $[\mathrm{Na} / \mathrm{Fe}] \leqslant-0.5$ abundances, which is contrary to Galactic GC stars. As was expected, $[\mathrm{Mg} / \mathrm{Fe}],[\mathrm{Si} / \mathrm{Fe}],[\mathrm{Ca} / \mathrm{Fe}]$, and $[\mathrm{Ti} / \mathrm{Fe}]$ are systematically lower than the Galactic counterparts, starting to decrease at around $[\mathrm{Fe} / \mathrm{H}] \simeq-2 .[\mathrm{Mn} / \mathrm{Fe}]$ shows a mirrow images of $[\alpha / F e]$, increasing at $[F e / H] \simeq-2$. All these strongly suggest a significant contribution of SNeIa at such early stage of chemical enrichment of Ursa Minor dSph. In one star $\operatorname{COS} 82([\mathrm{Fe} / \mathrm{H}]=-1.5)$, however, we have found a large excess of heavy neutron-capture elements with a general abundance pattern similar to the scaled solar system $r$-process abundance curve. This is a bit puzzling, because it means there is no hint of an $s$-process (i.e., AGB stars) contribution, even at $[\mathrm{Fe} / \mathrm{H}]=-1.5$, suggesting a peculiar nucleosynthetic history of the Ursa Minor dSph galaxy (Sadakane et al. (2004)).

\section{CMD Morphologies of $\mathrm{dSph} / \mathrm{dE} / \mathrm{dIrr}$ Galaxies}

CMD morphologies of dSphe/dEs clearly indicate that star formation had lasted 3.58.0 Gyrs in almost all $\mathrm{dSph} / \mathrm{dE}$ galaxies. We have studied the star formation histories of a sample of dwarf galaxies in the Local Group, via the analysis and modeling, with the mean of an evolutionary stellar population synthesis, of their CMDs (Ikuta, Arimoto, \& Jablonka (2005)). We have built a uniform set of CMDs derived from images taken with the Hubble Space Telescope. Our sample is composed of Andromeda I, Andromeda II, Andromeda III, Fornax, GR8, IC10, IC1613, Leo I, Leo II, Leo A, M32, NGC147, NGC185, NGC205, Pegasus, Phoenix, Sextans A, and WLM. We could distinguish several groups, based on their CMD morphology. The width of the RGB determines two different classes of objects, referenced as the Class I and II. Class I galaxies have RGBs narrower than the Class II galaxies. Based on the fraction of young $(<1 \mathrm{Gyr})$ or intermediate age $(1-5$ Gyr) stellar populations, the Class I and Class II are further divided into three different sub-classes from (a) to (c). By means of our CM diagram simulator, we show that the star formation histories of the Local Group dwarf galaxies can be simply described by two main parameters, the SFR and the length of the star formation. Galaxies grouped in the Class I have similar SFRs, regardless of their morphology. The variation of length of their star formation period explains their difference. This suggests a common origin for the early and late type dwarf galaxies. We find a correlation between the duration of the star formation in a given dwarf galaxy and its distance to M31 or the Milky Way; star formation lasted longer in galaxies at larger distance. This suggests that environmental effects play an important role in the evolution of dwarf galaxies and that strong tidal forces might well transform late-type dwarf galaxies into early-type ones. The Class II galaxies are characterised by SFRs stronger than what is necessary to reproduce the properties of the Class I objects (see figure captions of Figs.2-3). We find a possible correlation between the mass of the galaxies and their SFRs, ie, more massive galaxies tend to have much higher SFRs. This correlation might be the origin of the observed luminosity-metallicity relation in the Local Group dwarf galaxies. 

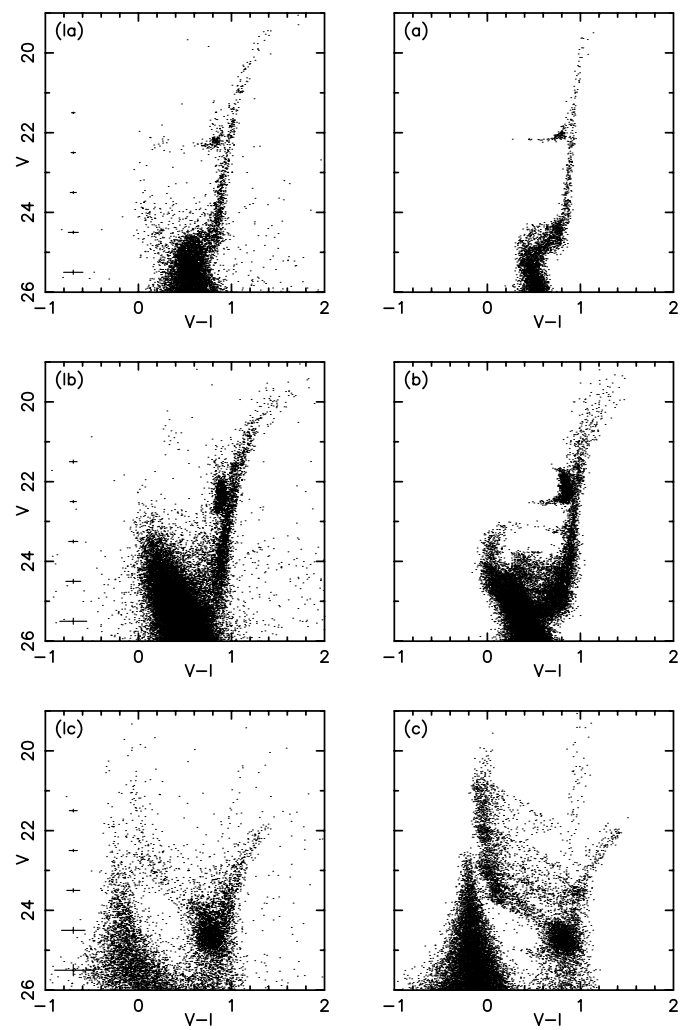

Figure 2. The observed (left side) and the best-fit simulated CM diagrams (right side, model a, b, c) of Leo II (Ia), Leo I (Ib), and Leo A (Ic). In the three cases, we adopted a galactic age of $12 \mathrm{Gyr}$, which is similar to the age of the Galactic globular clusters, and SFR $\epsilon=0.025 \epsilon \odot$ which we adopted for Draco, Sextans, and Ursa Minor dSphs. The length of the star formation period are $\Delta T_{\mathrm{SF}}=6.5,11$, and 12 Gyr for the Class Ia, Ib, and Ic, respectively.

\section{Line Strength Gradients in M32}

M32, a satelite dE of Andromeda galaxy, shows conspicuous gradients of age and metallicity. We have conducted long-slit spectroscopy of M32 with Subaru/FOCAS (Rose et al. (2005)). The spectra cover the Lick index red spectral region, as well as higher order Balmer lines in the blue. Spectra have been taken with the slit offset from the nucleus to avoid scattered-light contamination from the bright nucleus of M32. An analysis of numerous absorption features, particularly involving the $H \gamma$ and $H \beta$ Balmer lines, reveals that systematic radial trends are evident in the integrated spectrum of M32. Population synthesis models indicate a radial change in both the age and chemical composition of the light-weighted mean stellar population in M32, from the nucleus out to $33^{\prime \prime}$, i.e., an approximately 1.0 effective radius, $R_{e}$. Specifically, the light-weighted mean stellar population at $1 R_{e}$ is older by $\sim 3 \mathrm{Gyr}$ and more metal-poor by about -0.25 dex in $[\mathrm{Fe} / \mathrm{H}]$ than the central value of $\sim 4 \mathrm{Gyr}$ and $[\mathrm{Fe} / \mathrm{H}] \sim 0.0$ (Figs. $4-5$ ). This apparent population trend cannot be attributed to a varying contribution from either hot stars or emission-line contamination. The increase in age and decrease in metal abundance with radius are sufficiently well matched to explain the flat radial color profiles previously observed in $\mathrm{M} 32$. In addition, the ratio of $\mathrm{Mg}$ to $\mathrm{Fe}$ abundance, $[\mathrm{Mg} / \mathrm{Fe}]$, increases from about -0.25 in the nucleus to about -0.08 at $1 R_{e}$, ie, more significant and progressive contribution of SNeIa towards galaxy centre of M32. Natural explanation states that star 

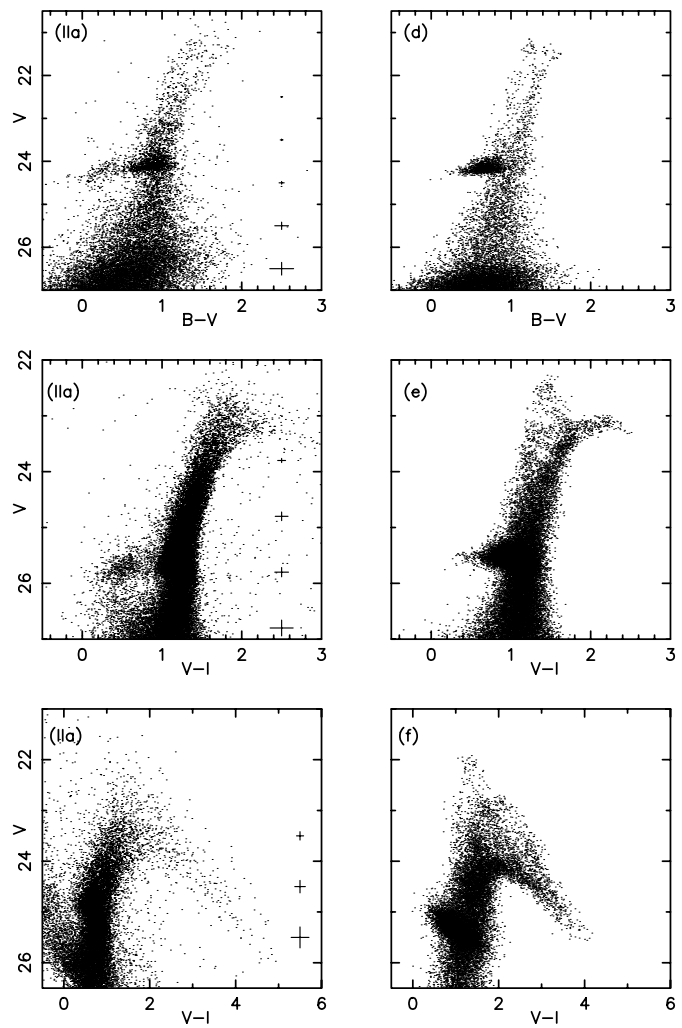

Figure 3. The observed (left side) and the best-fit simulated CM diagrams (right side, model d, e, f) of Andromeda II (IIa), NGC147 (IIa), and M32 (IIa). In the three cases, we adopted a galactic age of 12 Gyr. SFRs and time duration of star formatin period are $\left(\epsilon / \epsilon_{\odot}, \Delta T_{\mathrm{SF}} / \mathrm{Gyr}\right)=(0.05,4.5),(0.17,3.5)$ and $(0.50,8.0)$ for models $(\mathrm{d}),(\mathrm{e})$, and $(\mathrm{f})$, respectively.

formation in M32 lasted a few Gyr in whole part of the galaxy and then star forming region shirinks, but star formation continued till $\sim 8$ Gyr.

\section{Are Dwarf Galaxies Building Blocks?}

Dwarf galaxies might not be the smallest unit (building blocks) of galaxy formation. We have imaged the smallest dwarf irregular galaxy in the Local Group, Leo A, by using Subaru/Suprime-Cam (Vansevicius et al. (2004)). Leo A is extremely gas rich and possesses very low stellar mass and metallicity. It contains young stars as well as old stellar population. It has been suggested in that past that Leo A has not experienced any strong event of merger or interaction for at least a few Gyrs; thus it is a good target for study of quiescent galaxy evolution.

Figure 6 shows the radial profile of the RGB star surface number density $\left(\operatorname{arcmin}^{-2}\right)$ of Leo A which was constructed by integrating within elliptical $(b / a=0.60)$ rings of width. Five distinct radial profile zones are noticeable: (1) a crowded central part, $a=$ $0.0^{\prime}-2.0^{\prime}(2)$ an old exponential disc extending far beyond the previously estimated size of the galaxy (Mateo (1998)), $a=2.0^{\prime}-5.5^{\prime} ;(3)$ the discovered stellar component in Leo A, which we call "halo," $a=5.5^{\prime}-7.5^{\prime}$; (4) a sharp cutoff of the RGB star distribution coincident with the observed edge (Young \& Lo (1996)) and predicted cutoff 


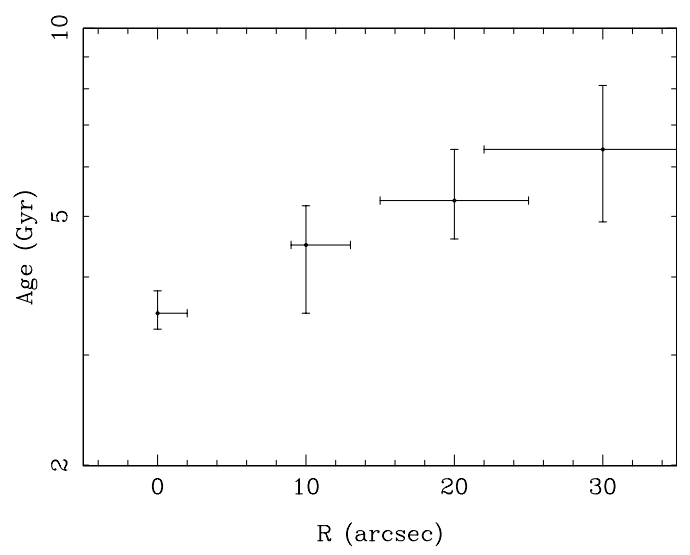

Figure 4. Spectroscopic age gradient of M32. Ages are derived from the robust age indicator $H_{\gamma_{\sigma}}$ defined by Vazdekis \& Arimoto (1999).

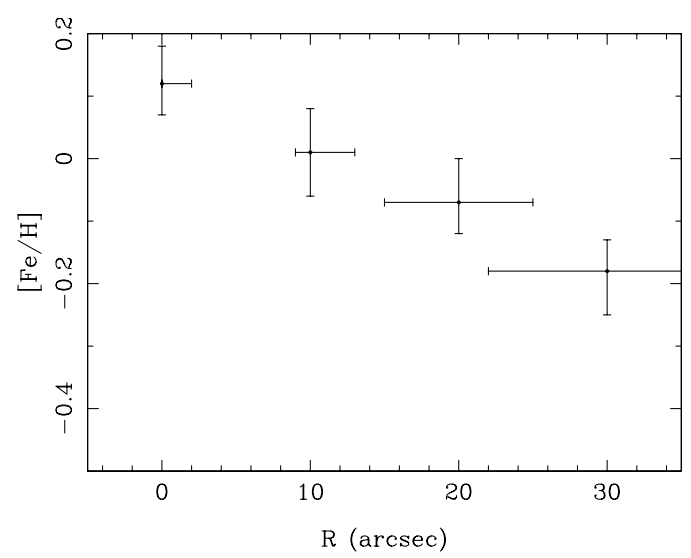

Figure 5. Spectroscopic metallicity gradient of M32. $[\mathrm{Fe} / \mathrm{H}]$ are derived from $\mathrm{Fe} 3$ index. Contrary to $\mathrm{Fe} 3$ feature, $\mathrm{Mg}_{2}$ index does not show any strong gradient, demonstrating very little gradient in $[M g / H]$ abundance.

of the H I envelope (Sternberg, McKee \& Wolfire (2002)), $a=7.5^{\prime}-8.0^{\prime}$; and (5) a sky background zone where we derived a number density of contaminants to the RGB stars, $a=8.0^{\prime}-12.0^{\prime}$. From the discovery of an old stellar halo and a sharp stellar edge as well as stellar and gaseous content, Leo A is found to resemble massive disc galaxies. This implies that galaxies of very low stellar mass are also able to develop complex structures.

Another evidence for complex early evolution of dSph galaxies is recently discovered by Tolstoy et al. (2004) who found evidence for the presence of two distinct ancient stellar components (both $\geqslant 10$ Gyr old) in the Sculptor dSph by using the ESO Wide Field Imager in conjunction with the VLT/FLAMES spectrograph. We find that two components are discernible in the spatial distribution of HB stars in our imaging and in the $[\mathrm{Fe} / \mathrm{H}]$ and $v_{\text {hel }}$ distributions for our large sample of spectroscopic measurements. Sculpter dSph is composed of a "metal-poor" component $([\mathrm{Fe} / \mathrm{H}]<-1.7)$ and a "metalrich" component $([\mathrm{Fe} / \mathrm{H}]>-1.7)$. The metal-poor stars are more spatially extended than the metal-rich stars, and they also appear to be kinematically distinct. Therefore, both Leo A (dIrr) and Sculpter (dSph) indicate that even these simplest of galaxies appear to have had a surprisingly complex early evolution. 


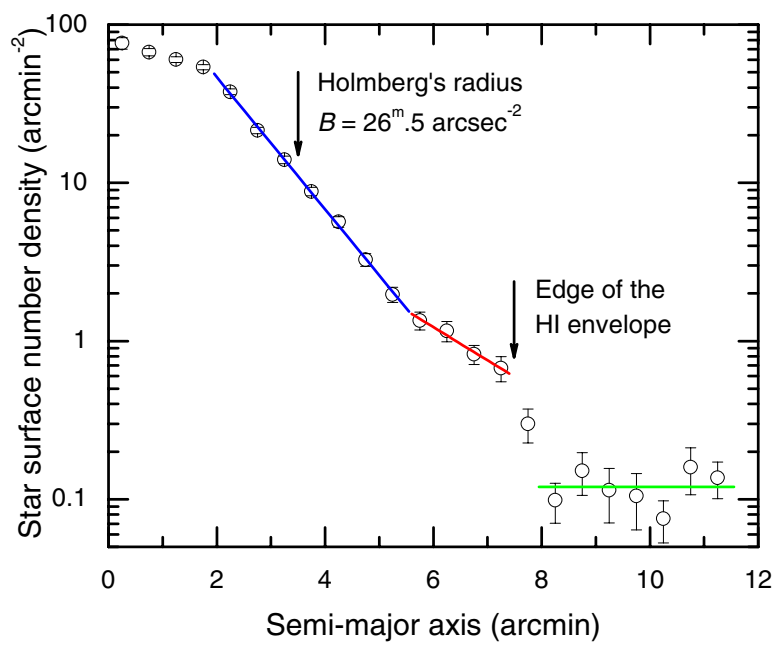

Figure 6. Radial profile of the RGB star surface number density in Leo A.

\section{Modeling Star Formation History of Dwarf Ellipticals}

To explain complex structure of the Sculpter dSph, we have performed cosmological simulations (Kawata \& Arimoto (2005)). Basic features of our model are described by Kawata \& Gibson (2003). In the simulation, dSph galaxies formed via hierarchical clustering, but stars formed from cold gas. Star formation started at $z=20$ and terminated by $z=4.8$, thus lasted only $\sim 1.0 \mathrm{Gyr}$, too short to expect significant contribution of SNeIa in chemical enrichment. Stars formed at the galaxy centre tend to form from metal-rich infall gas, which builds up the metallicity gradients. Infalling gas has larger rotational velocity and small velocity dispersion due to dissipative processes. The simulations can explain 1) the metallicity gradient and 2) the kinematic behaviours of stars at different distance from the galaxy centre, but it clearly fails to reproduce the observed abundance pattern of $[\alpha / F e]$ vs $[F e / H]$ which is now well established for most of $\mathrm{dSph}$ galaxies so far observed, requiring a significant improvement in the modeling $\mathrm{SFH}$ of dEs.

\section{Conclusions}

Modeling of star formation histories of dwarf ellipticals is not yet satisfactory. Cosmological numerical simulations can reproduce the complex features of chemical and kinematical properties of dSph galaxies, but fail to explain why SNeIa had contributed so sigificantly in a very early stage of chemical enrichment.

\section{Acknowledgements}

This work was financially supported in part by a Grant-in-Aid for the Scientific Research (No.16540223) by the Japanese Ministry of Education, Culture, Sports and Science. 


\section{References}

Ikuta, C., Arimoto, N., \& Jablonka, P. 2005, in preparation

Ikuta, C. \& Arimoto, N. 2002, A\&A A 391, 55

Kawata, D. \& Arimoto, N. 2005, in preparation

Kawata, D. \& Gibson, B.K. 2003, MNRAS 346, 135

Mateo, M. 1998, ARAESA 36, 435

Matteucci, F. \& Brocato, E. 1990, ApJ 365, 539

Matteucci, F. \& Greggio, L. 1986, A\&SA 154, 279

Rose, J.A., Arimoto, N., Caldwell, N., Schiavon, R., Vazdekis, A. \& Yamada, Y. 2005, AJ 129, 712

Sadakane, K., Arimoto, N., Aoki, W., Ohkubo, M., Ohnishi, K. \& Tajitsu, A. 2005, in preparation

Sadakane, K., Arimoto, N., Ikuta, C., Aoki, W., Jablonka, P. \& Tajitsu, A. 2004, PASJ 56, 1041

Shetrone, M.D., Côtè, P. \& Sargent, W.L.W. 2001, ApJ 548, 592

Sternberg, A., McKee, Ch. \& Wolfire, M.G. 2002, ApJS 143, 419

Tolstoy, E., et al. 2004, ApJL 617, L119

Vansevicius, V., Arimoto, N., Hasegawa, T. et al. 2004, ApJL 611, L93

Vazdekis, A. \& Arimoto, N. 1999, ApJ 525, 144

Young, L.M. \& Lo, K.Y. 1996, ApJ 462, 203

Yoshii, Y. \& Arimoto, N. 1987, A\&A A 188, 13

\section{Discussion}

LIN: The total heavy element content in $\mathrm{dSph}$ is comparable to the output of a few supernovae. Unless there is a significant preferential loss of supernovae ejecta, dSph may be over polluted by self contamination. However the formation of several generation of stars, with a metallicity dispersion, requires gas retention. How do you balance heavy element loss, gas retention, and protracted star formation process in your model?

Arimoto: Perhaps dSphs were more massive at the beginning, but lost substantial amount of mass later.

FERGuson: What is the explanation for the different widths of the RGB in your class Ia and IIa models? Is the narrow RGB in the Ia models simply a consequence of the wider distribution of age contradicting the color-spread that comes from metallicity? Or does the different charactor of winds in the Ia and IIa models also playing a role?

Arimoto: It is due to the age-metallicity degeneracy effect.

LISKER: In your sample of Virgo dEs, do you have nucleated and non-nucleated dEs, and if so, do you see differences in their spectral indices?

Arimoto: No, we do not see any difference.

SILVA: I have two comments: 1) Integrated light (features) is always luminosity-weighted. So, the mass driving the feature may be quite small. Note that due to age-metallicity degeneracy, this issue can affect "age" features and "metal" features. 2) When considering the larger cosmological context, remember that it is now clear that galaxies, supermassive black holes, and large scale structure are forming faster in the real universe than in current generator $\lambda \mathrm{CDM}$ based hierarchical merger simulations. In short, in the real universe, the clock is running fatser than in the simulated universe. 